\title{
Some New Volterra-Fredholm-Type Nonlinear Discrete Inequalities with Two Variables Involving Iterated Sums and Their Applications
}

\author{
Run Xu \\ Department of Mathematics, Qufu Normal University, Qufu, Shandong 273165, China \\ Correspondence should be addressed to Run Xu; xurun_2005@163.com
}

Received 27 April 2017; Accepted 13 August 2017; Published 27 September 2017

Academic Editor: Samir H. Saker

Copyright (C) 2017 Run Xu. This is an open access article distributed under the Creative Commons Attribution License, which permits unrestricted use, distribution, and reproduction in any medium, provided the original work is properly cited.

Some generalized discrete Volterra-Fredholm-type inequalities were developed, which can be used as effective tools in the qualitative analysis of the solution to difference equations.

\section{Introduction}

In recent years, various forms of inequalities played increasingly important roles in the study of quantitative properties of solutions of differential and integral equations [115]. Discrete inequalities, especially the discrete VolterraFredholm-type inequalities, have been applied to study the discrete equations widely. For example, see $[1-3,9-11]$ and the references therein. In this paper, some new VolterraFredholm-type discrete inequalities involving four iterated infinite sums were established. Furthermore, to illustrate the usefulness of the established results, some examples were provided for the studying of their solutions on the boundedness, uniqueness, and continuous dependence.

We design the needed symbols as follows:

(a) $N_{0}$ denotes the set of nonnegative integers and $Z$ denotes the set of integers, while $R$ denotes the set of real numbers $R_{+}=[0, \infty)$.

(b) Let $\Omega:=\left(\left[m_{0}, M\right] \times\left[n_{0}, N\right]\right) \cap Z^{2}$, where $m_{0}, n_{0} \in Z$, and $M, N \in Z \cup\{\infty\}$ are two constants.

(c) $K_{i}>0(i=1,2,3,4)$ are all constants, and $l_{1}, l_{2} \in Z$ are two constants.

(d) If $U$ is a lattice, then we denote the set of all $R$-valued functions on $U$ by $\wp(U)$ and denote the set of all $R_{+}$-valued functions on $U$ by $\wp_{+}(U)$. (e) For a function $g \in \wp_{+}(U)$, we have $\sum_{s=m_{0}}^{m_{1}} g(s)=0$ provided $m_{0}>m_{1}$.

We need the following lemmas in the discussions of our main results.

Lemma 1 (see [4]). Let $u(m, n) \in \wp_{+}(\Omega), b(s, t, m, n) \in$ $\wp_{+}\left(\Omega^{2}\right)$ be nondecreasing in the third variable; $k \geq 0$ is a constant. For $(m, n) \in \Omega$, if

$$
u(m, n) \leq k+\sum_{s=m_{0}}^{m-1} \sum_{t=n_{0}}^{n-1} b(s, t, m, n) u(s, t),
$$

then

$$
u(m, n) \leq k \exp \left\{\sum_{s=m_{0}}^{m-1} \sum_{t=n_{0}}^{n-1} b(s, t, m, n)\right\} .
$$

Lemma 2 (see [4]). Let $u(m, n), a(m, n), c(m, n) \in \wp_{+}(\Omega)$. If $a(m, n)$ is nondecreasing in the first variable, then, for $(m, n) \in$ $\Omega$,

$$
u(m, n) \leq a(m, n)+\sum_{s=m_{0}}^{m-1} c(s, n) u(s, n),
$$

then

$$
u(m, n) \leq a(m, n) \prod_{s=m_{0}}^{m-1}[1+c(s, n)] .
$$


Lemma 3 (see [5]). Let $a \geq 0, p \geq q \geq 0$, and $p \neq 0$; then, for any $K>0$,

$$
a^{q / p} \leq \frac{q}{p} K^{(q-p) / p} a+\frac{p-q}{p} K^{q / p} .
$$

\section{Main Results}

Theorem 4. Suppose that $u(m, n), a(m, n), b_{1}(m, n), b_{2}(m$, $n) \in \wp_{+}(\Omega), c_{i}(s, t, m, n), d_{i}(s, t, m, n), e_{i}(s, t, m, n), f_{j}(s, t, m$, $n), g_{j}(s, t, m, n), w_{j}(s, t, m, n) \in \wp_{+}\left(\Omega^{2}\right)$, and $p, q_{i}, r_{i}, h_{j}, v_{j}$ are nonnegative constants with $p \geq q_{i}>0, p \geq r_{i}>0(i=$ $\left.1,2, \ldots, l_{1}\right), p \geq h_{j}>0, p \geq v_{j}>0\left(j=1,2, \ldots, l_{2}\right)$, and $c_{i}, d_{i}, e_{i}, f_{j}, g_{j}, w_{j}$ being nondecreasing in the last two variables, $b_{1}(m, n)$ and $b_{2}(m, n)$ are also nondecreasing. If

$$
u^{p}(m, n) \leq a(m, n)+b_{1}(m, n)
$$

$$
\begin{aligned}
& \cdot \sum_{i=1}^{l_{1}} \sum_{s=m_{0}}^{m-1} \sum_{t=n_{0}}^{n-1}\left[c_{i}(s, t, m, n) u^{q_{i}}(s, t)+d_{i}(s, t, m, n)\right. \\
& \left.\cdot u^{r_{i}}(s, t)+e_{i}(s, t, m, n)\right]+b_{2}(m, n) \\
& \cdot \sum_{j=1}^{l_{2}} \sum_{s=m_{0}}^{M-1} \sum_{t=n_{0}}^{N-1}\left[f_{j}(s, t, m, n) u^{h_{j}}(s, t)\right. \\
& \left.+g_{j}(s, t, m, n) u^{v_{j}}(s, t)+w_{j}(s, t, m, n)\right],
\end{aligned}
$$

then, for $(m, n) \in \Omega$, we have

$$
\begin{aligned}
& u(m, n) \\
& \quad \leq\left\{a(m, n)+b(m, n) \frac{J(M, N)}{1-\lambda(M, N)} C(m, n)\right\}^{1 / p},
\end{aligned}
$$

provided that $\lambda(M, N)<1$, where

$$
\begin{aligned}
& b(m, n)=\max \left\{b_{1}(m, n), b_{2}(m, n)\right\}, \\
& C(m, n)=\exp \left\{\sum_{s=m_{0}}^{m-1} \sum_{t=n_{0}}^{n-1} B(s, t, m, n)\right\}, \\
& B(s, t, m, n)=\sum_{i=1}^{l_{1}}\left[c_{i}(s, t, m, n) \frac{q_{i}}{p} K_{1}^{\left(q_{i}-p\right) / p}+d_{i}(s, t, m, n) \frac{r_{i}}{p} K_{2}^{\left(r_{i}-p\right) / p}\right] b(s, t), \\
& J(m, n)=\sum_{i=1}^{l_{1}} \sum_{s=m_{0}}^{m-1} \sum_{t=n_{0}}^{n-1}\left\{c_{i}(s, t, m, n)\left[\frac{q_{i}}{p} K_{1}^{\left(q_{i}-p\right) / p} a(s, t)+\frac{p-q_{i}}{p} K_{1}^{q_{i} / p}\right]\right. \\
& \left.\quad+d_{i}(s, t, m, n)\left[\frac{r_{i}}{p} K_{2}^{\left(r_{i}-p\right) / p} a(s, t)+\frac{p-r_{i}}{p} K_{2}^{r_{i} / p}\right]+e_{i}(s, t, m, n)\right\} \\
& \quad+\sum_{j=1}^{l_{2}} \sum_{s=m_{0}}^{M-1} \sum_{t=n_{0}}^{N-1}\left\{f_{j}(s, t, m, n)\left[\frac{h_{j}}{p} K_{3}^{\left(h_{j}-p\right) / p} a(s, t)+\frac{p-h_{j}}{p} K_{3}^{h_{j} / p}\right]\right. \\
& \left.\quad+g_{j}(s, t, m, n)\left[\frac{v_{j}}{p} K_{4}^{\left(v_{j}-p\right) / p} a(s, t)+\frac{p-v_{j}}{p} K_{4}^{v_{j} / p}\right]+w_{j}(s, t, m, n)\right\}, \\
& \left.\quad m_{j}, n\right)=\sum_{j=1}^{l_{2}} \sum_{s=m_{0}}^{M-1} \sum_{t=n_{0}}^{N-1}\left[f_{j}(s, t, m, n) \frac{h_{j}}{p} K_{3}^{\left(h_{j}-p\right) / p}+g_{j}(s, t, m, n) \frac{v_{j}}{p} K_{4}^{\left(v_{j}-p\right) / p}\right] b(s, t) C(s, t) .
\end{aligned}
$$

Proof. Given $b(m, n)=\max \left\{b_{1}(m, n), b_{2}(m, n)\right\}$, for $(m, n) \in$ $\Omega$, we have

$$
\begin{aligned}
& u^{p}(m, n) \leq a(m, n)+b(m, n) \\
& \cdot \sum_{i=1}^{l_{1}} \sum_{s=m_{0}}^{m-1} \sum_{t=n_{0}}^{n-1}\left[c_{i}(s, t, m, n) u^{q_{i}}(s, t)\right.
\end{aligned}
$$

$$
\begin{aligned}
& \left.+d_{i}(s, t, m, n) u^{r_{i}}(s, t)+e_{i}(s, t, m, n)\right]+b(m, n) \\
& \cdot \sum_{j=1}^{l_{2}} \sum_{s=m_{0}}^{M-1} \sum_{t=n_{0}}^{N-1}\left[f_{j}(s, t, m, n) u^{h_{j}}(s, t)\right. \\
& \left.+g_{j}(s, t, m, n) u^{v_{j}}(s, t)+w_{j}(s, t, m, n)\right] .
\end{aligned}
$$

Define a function $z(m, n)$ by 


$$
\begin{aligned}
z(m, n)= & \sum_{i=1}^{l_{1}} \sum_{s=m_{0}}^{m-1} \sum_{t=n_{0}}^{n-1}\left[c_{i}(s, t, m, n) u^{q_{i}}(s, t)+d_{i}(s, t, m, n) u^{r_{i}}(s, t)+e_{i}(s, t, m, n)\right] \\
& +\sum_{j=1}^{l_{2}} \sum_{s=m_{0}}^{M-1} \sum_{t=n_{0}}^{N-1}\left[f_{j}(s, t, m, n) u^{h_{j}}(s, t)+g_{j}(s, t, m, n) u^{v_{j}}(s, t)+w_{j}(s, t, m, n)\right] .
\end{aligned}
$$

Then

$$
u^{p}(m, n) \leq a(m, n)+b(m, n) z(m, n),
$$

or

$$
u(m, n) \leq(a(m, n)+b(m, n) z(m, n))^{1 / p} .
$$

$$
\begin{aligned}
& z(m, n) \leq \sum_{i=1}^{l_{1}} \sum_{s=m_{0}}^{m-1} \sum_{t=n_{0}}^{n-1}\left\{c_{i}(s, t, m, n)\left[\frac{q_{i}}{p} K_{1}^{\left(q_{i}-p\right) / p}(a(s, t)+b(s, t) z(s, t))+\frac{p-q_{i}}{p} K_{1}^{q_{i} / p}\right]\right. \\
& \left.+d_{i}(s, t, m, n)\left[\frac{r_{i}}{p} K_{2}^{\left(r_{i}-p\right) / p}(a(s, t)+b(s, t) z(s, t))+\frac{p-r_{i}}{p} K_{2}^{r_{i} / p}\right]+e_{i}(s, t, m, n)\right\} \\
& +\sum_{j=1}^{l_{2}} \sum_{s=m_{0}}^{M-1} \sum_{t=n_{0}}^{N-1}\left\{f_{j}(s, t, m, n)\left[\frac{h_{j}}{p} K_{3}^{\left(h_{j}-p\right) / p}(a(s, t)+b(s, t) z(s, t))+\frac{p-h_{j}}{p} K_{3}^{h_{j} / p}\right]\right. \\
& \left.+g_{j}(s, t, m, n)\left[\frac{v_{j}}{p} K_{4}^{\left(v_{j}-p\right) / p}(a(s, t)+b(s, t) z(s, t))+\frac{p-v_{j}}{p} K_{4}^{v_{j} / p}\right]+w_{j}(s, t, m, n)\right\}=R(m, n) \\
& +\sum_{i=1}^{l_{1}} \sum_{s=m_{0}}^{m-1} \sum_{t=n_{0}}^{n-1}\left[c_{i}(s, t, m, n) \frac{q_{i}}{p} K_{1}^{\left(q_{i}-p\right) / p}+d_{i}(s, t, m, n) \frac{r_{i}}{p} K_{2}^{\left(r_{i}-p\right) / p}\right] b(s, t) z(s, t),
\end{aligned}
$$

where

$$
R(m, n)=J(m, n)+\sum_{j=1}^{l_{2}} \sum_{s=m_{0}}^{M-1} \sum_{t=n_{0}}^{N-1}\left[f_{j}(s, t, m, n) \frac{h_{j}}{p} K_{3}^{\left(h_{j}-p\right) / p}+g_{j}(s, t, m, n) \frac{v_{j}}{p} K_{4}^{\left(v_{j}-p\right) / p}\right] b(s, t) z(s, t),
$$

and $J(m, n)$ is defined in (11). Then, using that $R(m, n)$ is nondecreasing in every variable, we get

$$
\begin{aligned}
z(m, n) & \leq R(M, N)+\sum_{i=1}^{l_{1}} \sum_{s=m_{0}}^{m-1} \sum_{t=n_{0}}^{n-1}\left[c_{i}(s, t, m, n) \frac{q_{i}}{p} K_{1}^{\left(q_{i}-p\right) / p}+d_{i}(s, t, m, n) \frac{r_{i}}{p} K_{2}^{\left(r_{i}-p\right) / p}\right] b(s, t) z(s, t) \\
& =R(M, N)+\sum_{s=m_{0}}^{m-1} \sum_{t=n_{0}}^{n-1} B(s, t, m, n) z(s, t),
\end{aligned}
$$

where $B(s, t, m, n)$ is defined in (10). 
Since $b(m, n)$ is nondecreasing and $c_{i}(s, t, m, n), d_{i}(s, t$, $m, n)$ are nondecreasing in the last two variables, then $B(s, t, m, n)$ is also nondecreasing in the last two variables, and, by Lemma 1 and (19), we get

$$
\begin{aligned}
z(m, n) & \leq R(M, N) \exp \left\{\sum_{s=m_{0}}^{m-1} \sum_{t=n_{0}}^{n-1} B(s, t, m, n)\right\} \\
& =R(M, N) C(m, n),
\end{aligned}
$$

where $C(m, n)$ is defined in (9). Considering the definition of $R(m, n)$ and (20), we have

$$
\begin{aligned}
& R(M, N)=J(M, N) \\
& +\sum_{j=1}^{l_{2}} \sum_{s=m_{0}}^{M-1} \sum_{t=n_{0}}^{N-1}\left[f_{j}(s, t, M, N) \frac{h_{j}}{p} K_{3}^{\left(h_{j}-p\right) / p}\right. \\
& \left.+g_{j}(s, t, M, N) \frac{v_{j}}{p} K_{4}^{\left(v_{j}-p\right) / p}\right] b(s, t) z(s, t) \leq J(M, \\
& N)+R(M, N) \\
& \quad+\sum_{j=1}^{l_{2}} \sum_{s=m_{0}}^{M-1} \sum_{t=n_{0}}^{N-1}\left[f_{j}(s, t, M, N) \frac{h_{j}}{p} K_{3}^{\left(h_{j}-p\right) / p}\right. \\
& \left.+g_{j}(s, t, M, N) \frac{v_{j}}{p} K_{4}^{\left(v_{j}-p\right) / p}\right] b(s, t) C(s, t) \\
& \quad=J(M, N)+R(M, N) \lambda(M, N),
\end{aligned}
$$

where $\lambda(m, n)$ is defined in (12). Then,

$$
R(M, N) \leq \frac{J(M, N)}{1-\lambda(M, N)} .
$$

Combining (20) and (22), we deduce

$$
z(m, n) \leq \frac{J(M, N)}{1-\lambda(M, N)} C(m, n),
$$

where $C(m, n), \lambda(m, n)$ are defined in (9) and (12). result.

Then, combining (16) and (23), we obtain the desired

Corollary 5. Let $r_{1 i}(m, n), d_{1 i}(m, n), c_{1 i}(m, n), e_{1 i}(m, n) \quad \epsilon$ $\wp_{+}(\Omega), \quad\left(i=1,2, \ldots, l_{1}\right), f_{1 j}(m, n), g_{1 j}(m, n), w_{1 j}(m, n)$, $r_{2 j}(m, n) \in \wp_{+}(\Omega),\left(j=1,2, \ldots, l_{2}\right), r_{1 i}(m, n), r_{2 j}(m, n)$, $b_{1}(m, n)$ and $b_{2}(m, n)$ be nondecreasing in every variable. $u(m, n), a(m, n), b_{1}(m, n), b_{2}(m, n), p, q_{i}, r_{i}, h_{j}, v_{j}$ are defined as in Theorem 4. If

$$
\begin{aligned}
& u^{p}(m, n) \leq a(m, n)+b_{1}(m, n) \sum_{i=1}^{l_{1}} r_{1 i}(m, n) \\
& \cdot \sum_{s=m_{0}}^{m-1} \sum_{t=n_{0}}^{n-1}\left[c_{1 i}(s, t) u^{q_{i}}(s, t)+d_{1 i}(s, t) u^{r_{i}}(s, t)\right.
\end{aligned}
$$

$$
\begin{aligned}
& \left.+e_{1 i}(s, t)\right]+b_{2}(m, n) \sum_{j=1}^{l_{2}} r_{2 j}(m, n) \\
& \cdot \sum_{s=m_{0}}^{M-1} \sum_{t=n_{0}}^{N-1}\left[f_{1 j}(s, t) u^{h_{j}}(s, t)\right. \\
& \left.+g_{1 j}(s, t) u^{v_{j}}(s, t)+w_{1 j}(s, t)\right],
\end{aligned}
$$

then, for $(m, n) \in \Omega$, we have

$u(m, n)$

$$
\leq\left\{a(m, n)+b(m, n) \frac{J(M, N)}{1-\lambda(M, N)} C(m, n)\right\}^{1 / p},
$$

provided that $\lambda(M, N)<1$, where

$b(m, n)=\max \left\{b_{1}(m, n), b_{2}(m, n)\right\}$,

$C(m, n)=\exp \left\{\sum_{s=m_{0}}^{m-1} \sum_{t=n_{0}}^{n-1} B(s, t, m, n)\right\}$,

$B(s, t, m, n)=\sum_{i=1}^{l_{1}} r_{1 i}(m, n)\left[c_{1 i}(s, t) \frac{q_{i}}{p} K_{1}^{\left(q_{i}-p\right) / p}\right.$

$\left.+d_{1 i}(s, t) \frac{r_{i}}{p} K_{2}^{\left(r_{i}-p\right) / p}\right] b(s, t)$

$J(m, n)=\sum_{i=1}^{l_{1}} r_{1 i}(m, n) \sum_{s=m_{0}}^{m-1} \sum_{t=n_{0}}^{n-1}\left\{c_{1 i}(s, t)\right.$

$\cdot\left[\frac{q_{i}}{p} K_{1}^{\left(q_{i}-p\right) / p} a(s, t)+\frac{p-q_{i}}{p} K_{1}^{q_{i} / p}\right]+d_{1 i}(s, t)$

$\left.\cdot\left[\frac{r_{i}}{p} K_{2}^{\left(r_{i}-p\right) / p} a(s, t)+\frac{p-r_{i}}{p} K_{2}^{r_{i} / p}\right]+e_{1 \mathrm{i}}(s, t)\right\}$

$+\sum_{j=1}^{l_{2}} r_{2 j}(m, n) \sum_{s=m_{0}}^{M-1} \sum_{t=n_{0}}^{N-1}\left\{f_{1 j}(s, t)\right.$

$\cdot\left[\frac{h_{j}}{p} K_{3}^{\left(h_{j}-p\right) / p} a(s, t)+\frac{p-h_{j}}{p} K_{3}^{h_{j} / p}\right]+g_{1 j}(s, t)$

$\left.\cdot\left[\frac{v_{j}}{p} K_{4}^{\left(v_{j}-p\right) / p} a(s, t)+\frac{p-v_{j}}{p} K_{4}^{v_{j} / p}\right]+w_{1 j}(s, t)\right\}$,

$\lambda(m, n)=\sum_{j=1}^{l_{2}} r_{2 j}(m, n) \sum_{s=m_{0}}^{M-1} \sum_{t=n_{0}}^{N-1}\left[f_{1 j}(s, t) \frac{h_{j}}{p} K_{3}^{\left(h_{j}-p\right) / p}\right.$

$\left.+g_{1 j}(s, t) \frac{v_{j}}{p} K_{4}^{\left(v_{j}-p\right) / p}\right] b(s, t) C(s, t)$.

The proof of Corollary 5 can be completed by setting $c_{i}(s, t, m, n)=r_{1 i}(m, n) c_{1 i}(s, t), d_{i}(s, t, m, n)=r_{1 i}(m, n) d_{1 i}(s$, $t), e_{i}(s, t, m, n)=r_{1 i}(m, n) e_{1 i}(s, t), f_{j}(s, t, m, n)=r_{2 j}(m$, 
n) $f_{1 j}(s, t), g_{j}(s, t, m, n)=r_{2 j}(m, n) g_{1 j}(s, t), w_{j}(s, t, m, n)=$ $r_{2 j}(m, n) w_{1 j}(s, t)$ in Theorem 4 .

Letting $p=1$, we get the following corollary.
Corollary 6. Let $u(m, n), a(m, n), b_{1}(m, n), b_{2}(m, n), c_{i}(s, t, m$, $n), d_{i}(s, t, m, n), e_{i}(s, t, m, n), f_{j}(s, t, m, n), g_{j}(s, t, m, n), w_{j}(s$, $t, m, n)$ be defined as in Theorem 4 . If

$$
\begin{aligned}
u(m, n) \leq & a(m, n)+b_{1}(m, n) \sum_{i=1}^{l_{1}} \sum_{s=m_{0}}^{m-1} \sum_{t=n_{0}}^{n-1}\left[c_{i}(s, t, m, n) u(s, t)+d_{i}(s, t, m, n) u(s, t)+e_{i}(s, t, m, n)\right] \\
& +b_{2}(m, n) \sum_{j=1}^{l_{2}} \sum_{s=m_{0}}^{M-1} \sum_{t=n_{0}}^{N-1}\left[f_{j}(s, t, m, n) u(s, t)+g_{j}(s, t, m, n) u(s, t)+w_{j}(s, t, m, n)\right],
\end{aligned}
$$

then, for $(m, n) \in \Omega$, we have provided that $\lambda(M, N)<1$, where

$$
u(m, n) \leq a(m, n)+b(m, n) \frac{J(M, N)}{1-\lambda(M, N)} C(m, n),
$$

$$
\begin{aligned}
b(m, n)= & \max \left\{b_{1}(m, n), b_{2}(m, n)\right\}, \\
C(m, n)= & \exp \left\{\sum_{s=m_{0}}^{m-1} \sum_{t=n_{0}}^{n-1} B(s, t, m, n)\right\}, \\
B(s, t, m, n)= & \sum_{i=1}^{l_{1}}\left[c_{i}(s, t, m, n)+d_{i}(s, t, m, n)\right] b(s, t), \\
J(m, n)= & \sum_{i=1}^{l_{1}} \sum_{s=m_{0}}^{m-1} \sum_{t=n_{0}}^{n-1}\left\{\left[c_{i}(s, t, m, n)+d_{i}(s, t, m, n)\right] a(s, t)+e_{i}(s, t, m, n)\right\} \\
& +\sum_{j=1}^{l_{2}} \sum_{s=m_{0}}^{M-1} \sum_{t=n_{0}}^{N-1}\left\{\left[f_{j}(s, t, m, n)+g_{j}(s, t, m, n)\right] a(s, t)+w_{j}(s, t, m, n)\right\}, \\
\lambda(m, n)= & \sum_{j=1}^{l_{2}} \sum_{s=m_{0}}^{M-1} \sum_{t=n_{0}}^{N-1}\left[f_{j}(s, t, m, n)+g_{j}(s, t, m, n)\right] b(s, t) C(s, t) .
\end{aligned}
$$

Theorem 7. Let $\varphi(m, n) \in \wp_{+}(\Omega), u(m, n), a(m, n), b_{1}(m, n)$, $b_{2}(m, n), c_{i}(s, t, m, n), d_{i}(s, t, m, n), e_{i}(s, t, m, n), f_{j}(s, t, m, n)$, $g_{j}(s, t, m, n), w_{j}(s, t, m, n), p, q_{i}, r_{i}, h_{j}, v_{j}$ be defined as in Theorem 4. Assume that $a(m, n)$ is nondecreasing in the first variable. If

$$
\begin{aligned}
& u^{p}(m, n) \leq a(m, n)+\sum_{s=m_{0}}^{m-1} \varphi(s, n) u^{p}(s, n)+b_{1}(m, n) \\
& \quad \cdot \sum_{i=1}^{l_{1}} \sum_{s=m_{0}}^{m-1} \sum_{t=n_{0}}^{n-1}\left[c_{i}(s, t, m, n) u^{q_{i}}(s, t)\right. \\
& \left.+d_{i}(s, t, m, n) u^{r_{i}}(s, t)+e_{i}(s, t, m, n)\right]+b_{2}(m, n) \\
& \quad \cdot \sum_{j=1}^{l_{2}} \sum_{s=m_{0}}^{M-1} \sum_{t=n_{0}}^{N-1}\left[f_{j}(s, t, m, n)\right.
\end{aligned}
$$

$$
\left.\cdot u^{h_{j}}(s, t)+g_{j}(s, t, m, n) u^{v_{j}}(s, t)+w_{j}(s, t, m, n)\right],
$$

then, for $(m, n) \in \Omega$, we have

$$
\begin{aligned}
& u(m, n) \\
& \quad \leq\left\{\left[a(m, n)+b(m, n) \frac{\widetilde{J}(M, N)}{1-\tilde{\lambda}(M, N)} \widetilde{C}(m, n)\right]\right. \\
& \cdot \widetilde{\varphi}(m, n)\}^{1 / p},
\end{aligned}
$$

provided that $\tilde{\lambda}(M, N)<1$, where 


$$
\begin{aligned}
& \tilde{\varphi}(m, n)=\prod_{s=m_{0}}^{m-1}[1+\varphi(s, n)] \\
& b(m, n)=\max \left\{b_{1}(m, n), b_{2}(m, n)\right\}, \\
& \widetilde{c}_{i}(s, t, m, n)=c_{i}(s, t, m, n)(\widetilde{\varphi}(s, t))^{q_{i} / p} \text {, } \\
& \widetilde{d}_{i}(s, t, m, n)=d_{i}(s, t, m, n)(\widetilde{\varphi}(s, t))^{r_{i} / p}, \quad i=1,2, \ldots, l_{1}, \\
& \tilde{f}_{j}(s, t, m, n)=f_{j}(s, t, m, n)(\widetilde{\varphi}(s, t))^{h_{j} / p}, \\
& \tilde{g}_{j}(s, t, m, n)=g_{j}(s, t, m, n)(\widetilde{\varphi}(s, t))^{v_{j} / p}, \quad j=1,2, \ldots, l_{2}, \\
& \widetilde{C}(m, n)=\exp \left\{\sum_{s=m_{0}}^{m-1} \sum_{t=n_{0}}^{n-1} \widetilde{B}(s, t, m, n)\right\}, \\
& \widetilde{B}(s, t, m, n)=\sum_{i=1}^{l_{1}}\left[\widetilde{c}_{i}(s, t, m, n) \frac{q_{i}}{p} K_{1}^{\left(q_{i}-p\right) / p}+\tilde{d}_{i}(s, t, m, n) \frac{r_{i}}{p} K_{2}^{\left(r_{i}-p\right) / p}\right] b(s, t), \\
& \tilde{J}(m, n)=\sum_{i=1}^{l_{1}} \sum_{s=m_{0}}^{m-1} \sum_{t=n_{0}}^{n-1}\left\{\widetilde{c}_{i}(s, t, m, n)\left[\frac{q_{i}}{p} K_{1}^{\left(q_{i}-p\right) / p} a(s, t)+\frac{p-q_{i}}{p} K_{1}^{q_{i} / p}\right]+\tilde{d}_{i}(s, t, m, n)\left[\frac{r_{i}}{p} K_{2}^{\left(r_{i}-p\right) / p} a(s, t)+\frac{p-r_{i}}{p}\right.\right. \\
& \left.\left.\cdot K_{2}^{r_{i} / p}\right]+e_{i}(s, t, m, n)\right\}+\sum_{j=1}^{l_{2}} \sum_{s=m_{0}}^{M-1} \sum_{t=n_{0}}^{N-1}\left\{\tilde{f}_{j}(s, t, m, n)\left[\frac{h_{j}}{p} K_{3}^{\left(h_{j}-p\right) / p} a(s, t)+\frac{p-h_{j}}{p} K_{3}^{h_{j} / p}\right]\right. \\
& \left.+\widetilde{g}_{j}(s, t, m, n)\left[\frac{v_{j}}{p} K_{4}^{\left(v_{j}-p\right) / p} a(s, t)+\frac{p-v_{j}}{p} K_{4}^{v_{j} / p}\right]+w_{j}(s, t, m, n)\right\}, \\
& \tilde{\lambda}(m, n)=\sum_{j=1}^{l_{2}} \sum_{s=m_{0}}^{M-1} \sum_{t=n_{0}}^{N-1}\left[\tilde{f}_{j}(s, t, m, n) \frac{h_{j}}{p} K_{3}^{\left(h_{j}-p\right) / p}+\widetilde{g}_{j}(s, t, m, n) \frac{v_{j}}{p} K_{4}^{\left(v_{j}-p\right) / p}\right] b(s, t) \widetilde{C}(s, t) .
\end{aligned}
$$

Proof. Given $b(m, n)=\max \left\{b_{1}(m, n), b_{2}(m, n)\right\}$, for $(m, n) \in$ $\Omega$, we have

$$
\begin{aligned}
& u^{p}(m, n) \leq a(m, n)+\sum_{s=m_{0}}^{m-1} \varphi(s, n) u^{p}(s, n)+b(m, n) \\
& \quad \cdot \sum_{i=1}^{l_{1}} \sum_{s=m_{0}}^{m-1} \sum_{t=n_{0}}^{n-1}\left[c_{i}(s, t, m, n) u^{q_{i}}(s, t)\right. \\
& \left.+d_{i}(s, t, m, n) u^{r_{i}}(s, t)+e_{i}(s, t, m, n)\right]+b(m, n) \\
& \quad \cdot \sum_{j=1}^{l_{2}} \sum_{s=m_{0}}^{M-1} \sum_{t=n_{0}}^{N-1}\left[f_{j}(s, t, m, n)\right. \\
& \left.\cdot u^{h_{j}}(s, t)+g_{j}(s, t, m, n) u^{v_{j}}(s, t)+w_{j}(s, t, m, n)\right] .
\end{aligned}
$$

Define function $\widetilde{z}(m, n)$ by

$$
\begin{aligned}
& \tilde{z}(m, n)=a(m, n)+b(m, n) \\
& \quad \cdot \sum_{i=1}^{l_{1}} \sum_{s=m_{0}}^{m-1} \sum_{t=n_{0}}^{n-1}\left[c_{i}(s, t, m, n) u^{q_{i}}(s, t)\right.
\end{aligned}
$$

$$
\begin{aligned}
& \left.+d_{i}(s, t, m, n) u^{r_{i}}(s, t)+e_{i}(s, t, m, n)\right]+b(m, n) \\
& \cdot \sum_{j=1}^{l_{2}} \sum_{s=m_{0}}^{M-1} \sum_{t=n_{0}}^{N-1}\left[f_{j}(s, t, m, n) u^{h_{j}}(s, t)\right. \\
& \left.+g_{j}(s, t, m, n) u^{v_{j}}(s, t)+w_{j}(s, t, m, n)\right] .
\end{aligned}
$$

Then,

$$
u^{p}(m, n) \leq \widetilde{z}(m, n)+\sum_{s=m_{0}}^{m-1} \varphi(s, n) u^{p}(s, n) .
$$

Clearly $z(m, n)$ is nondecreasing in the first variable. Then, by Lemma 2, we get

$$
\begin{aligned}
u^{p}(m, n) & \leq \widetilde{z}(m, n) \prod_{s=m_{0}}^{m-1}[1+\varphi(s, n)] \\
& =\widetilde{z}(m, n) \widetilde{\varphi}(m, n),
\end{aligned}
$$

where $\tilde{\varphi}(m, n)$ is defined in (32). Define function 


$$
\begin{aligned}
v(m, n)= & \sum_{i=1}^{l_{1}} \sum_{s=m_{0}}^{m-1} \sum_{t=n_{0}}^{n-1}\left[c_{i}(s, t, m, n) u^{q_{i}}(s, t)+d_{i}(s, t, m, n) u^{r_{i}}(s, t)+e_{i}(s, t, m, n)\right] \\
& +\sum_{j=1}^{l_{2}} \sum_{s=m_{0}}^{M-1} \sum_{t=n_{0}}^{N-1}\left[f_{j}(s, t, m, n) u^{h_{j}}(s, t)+g_{j}(s, t, m, n) u^{v_{j}}(s, t)+w_{j}(s, t, m, n)\right] .
\end{aligned}
$$

From (40), we get

$$
\widetilde{z}(m, n)=a(m, n)+b(m, n) v(m, n) .
$$

Then (42) becomes

$$
u(m, n) \leq\{[a(m, n)+b(m, n) v(m, n)] \widetilde{\varphi}(m, n)\}^{1 / p} .
$$

(44) By (45) and Lemma 3, from (43), we have

$$
\begin{aligned}
& v(m, n) \leq \sum_{i=1}^{l_{1}} \sum_{s=m_{0}}^{m-1} \sum_{t=n_{0}}^{n-1}\left\{c_{i}(s, t, m, n)(\widetilde{\varphi}(s, t))^{q_{i} / p}\left[\frac{q_{i}}{p} K_{1}^{\left(q_{i}-p\right) / p}(a(s, t)+b(s, t) v(s, t))+\frac{p-q_{i}}{p} K_{1}^{q_{i} / p}\right]\right. \\
& \left.+d_{i}(s, t, m, n)(\widetilde{\varphi}(s, t))^{r_{i} / p}\left[\frac{r_{i}}{p} K_{2}^{\left(r_{i}-p\right) / p}(a(s, t)+b(s, t) v(s, t))+\frac{p-r_{i}}{p} K_{2}^{r_{i} / p}\right]+e_{i}(s, t, m, n)\right\} \\
& \quad+\sum_{j=1}^{l_{2}} \sum_{s=m_{0}}^{M-1} \sum_{t=n_{0}}^{N-1}\left\{f_{j}(s, t, m, n)(\widetilde{\varphi}(s, t))^{h_{j} / p}\left[\frac{h_{j}}{p} K_{3}^{\left(h_{j}-p\right) / p}(a(s, t)+b(s, t) v(s, t))+\frac{p-h_{j}}{p} K_{3}^{h_{j} / p}\right]\right. \\
& \left.+g_{j}(s, t, m, n)(\widetilde{\varphi}(s, t))^{v_{j} / p}\left[\frac{v_{j}}{p} K_{4}^{\left(v_{j}-p\right) / p}(a(s, t)+b(s, t) v(s, t))+\frac{p-v_{j}}{p} K_{4}^{v_{j} / p}\right]+w_{j}(s, t, m, n)\right\}=\widetilde{R}(m, n) \\
& \quad+\sum_{i=1}^{l_{1}} \sum_{s=m_{0}}^{m-1} \sum_{t=n_{0}}^{n-1}\left[\widetilde{c}_{i}(s, t, m, n) \frac{q_{i}}{p} K_{1}^{\left(q_{i}-p\right) / p}+\widetilde{d}_{i}(s, t, m, n) \frac{r_{i}}{p} K_{2}^{\left(r_{i}-p\right) / p}\right] b(s, t) v(s, t),
\end{aligned}
$$

where

$$
\widetilde{R}(m, n)=\widetilde{J}(m, n)+\sum_{j=1}^{l_{2}} \sum_{s=m_{0}}^{M-1} \sum_{t=n_{0}}^{N-1}\left[\tilde{f}_{j}(s, t, m, n) \frac{h_{j}}{p} K_{3}^{\left(h_{j}-p\right) / p}+\widetilde{g}_{j}(s, t, m, n) \frac{v_{j}}{p} K_{4}^{\left(v_{j}-p\right) / p}\right] b(s, t) v(s, t),
$$

$\widetilde{c}_{i}, \widetilde{d}_{i}, \widetilde{f}_{j}, \widetilde{g}_{j}$ and $\widetilde{J}(m, n)$ are defined in (34) and (37), respectively.

Similar to the process of (17)-(23), we deduce that

$$
v(m, n) \leq \frac{\widetilde{J}(M, N)}{1-\widetilde{\lambda}(M, N)} \widetilde{C}(m, n),
$$

where $\widetilde{C}(m, n), \widetilde{\lambda}(m, n)$ are defined in (35) and (38).

Combining (45) and (48), we get the desired result.

Theorem 8. Let $u(m, n), a(m, n), b_{1}(m, n), b_{2}(m, n), c_{i}(s, t, m$ $n), d_{i}(s, t, m, n), e_{i}(s, t, m, n), f_{j}(s, t, m, n), g_{j}(s, t, m, n), w_{j}(s$, $t, m, n), p, q_{i}, r_{i}, h_{j}, v_{j}$ be defined as in Theorem 4. $H_{j}, L_{j}$ : $\Omega \times R_{+} \rightarrow R_{+}\left(j=1,2, \ldots, l_{2}\right)$ satisfies $0 \leq H_{j}(m, n, u)-$ $H_{j}(m, n, v) \leq L_{j}(m, n, v)(u-v)$ for $u \geq v \geq 0$. If

$$
u^{p}(m, n) \leq a(m, n)+b_{1}(m, n)
$$

$$
\begin{aligned}
& \cdot \sum_{i=1}^{l_{1}} \sum_{s=m_{0}}^{m-1} \sum_{t=n_{0}}^{n-1}\left[c_{i}(s, t, m, n) u^{q_{i}}(s, t)\right. \\
& \left.+d_{i}(s, t, m, n) u^{r_{i}}(s, t)+e_{i}(s, t, m, n)\right]+b_{2}(m, n) \\
& \cdot \sum_{j=1}^{l_{2}} \sum_{s=m_{0}}^{M-1} \sum_{t=n_{0}}^{N-1}\left[f_{j}(s, t, m, n) H_{j}\left(s, t, u^{h_{j}}(s, t)\right)\right. \\
& \left.+g_{j}(s, t, m, n) H_{j}\left(s, t, u^{v_{j}}(s, t)\right)+w_{j}(s, t, m, n)\right],
\end{aligned}
$$

then, for $(m, n) \in \Omega$, we have

$u(m, n)$

$$
\leq\left\{a(m, n)+b(m, n) \frac{\bar{J}(M, N)}{1-\bar{\lambda}(M, N)} \bar{C}(m, n)\right\}^{1 / p},
$$


provided that $\bar{\lambda}(M, N)<1$, where

$$
\begin{aligned}
& b(m, n)=\max \left\{b_{1}(m, n), b_{2}(m, n)\right\}, \\
& \bar{C}(m, n)=\exp \left\{\sum_{s=m_{0}}^{m-1} \sum_{t=n_{0}}^{n-1} \bar{B}(s, t, m, n)\right\} \text {, } \\
& \bar{B}(s, t, m, n)=\sum_{i=1}^{l_{1}}\left[c_{i}(s, t, m, n) \frac{q_{i}}{p} K_{1}^{\left(q_{i}-p\right) / p}+d_{i}(s, t, m, n) \frac{r_{i}}{p} K_{2}^{\left(r_{i}-p\right) / p}\right] b(s, t), \\
& \bar{J}(m, n)=\sum_{i=1}^{l_{1}} \sum_{s=m_{0}}^{m-1} \sum_{t=n_{0}}^{n-1}\left\{c_{i}(s, t, m, n)\left[\frac{q_{i}}{p} K_{1}^{\left(q_{i}-p\right) / p} a(s, t)+\frac{p-q_{i}}{p} K_{1}^{q_{i} / p}\right]\right. \\
& \left.+d_{i}(s, t, m, n)\left[\frac{r_{i}}{p} K_{2}^{\left(r_{i}-p\right) / p} a(s, t)+\frac{p-r_{i}}{p} K_{2}^{r_{i} / p}\right]+e_{i}(s, t, m, n)\right\} \\
& +\sum_{j=1}^{l_{2}} \sum_{s=m_{0}}^{M-1} \sum_{t=n_{0}}^{N-1}\left\{f_{j}(s, t, m, n) H_{j}\left[s, t, \frac{h_{j}}{p} K_{3}^{\left(h_{j}-p\right) / p} a(s, t)+\frac{p-h_{j}}{p} K_{3}^{h_{j} / p}\right]\right. \\
& \left.+g_{j}(s, t, m, n) H_{j}\left[s, t, \frac{v_{j}}{p} K_{4}^{\left(v_{j}-p\right) / p} a(s, t)+\frac{p-v_{j}}{p} K_{4}^{v_{j} / p}\right]+w_{j}(s, t, m, n)\right\}, \\
& \bar{f}_{j}(s, t, m, n)=f_{j}(s, t, m, n) L_{j}\left(s, t, \frac{h_{j}}{p} K_{3}^{\left(h_{j}-p\right) / p} a(s, t)+\frac{p-h_{j}}{p} K_{3}^{h_{j} / p}\right), \quad j=1,2, \ldots, l_{2}, \\
& \bar{g}_{j}(s, t, m, n)=g_{j}(s, t, m, n) L_{j}\left(s, t, \frac{v_{j}}{p} K_{4}^{\left(v_{j}-p\right) / p} a(s, t)+\frac{p-v_{j}}{p} K_{4}^{v_{j} / p}\right), \quad j=1,2, \ldots, l_{2}, \\
& \bar{\lambda}(m, n)=\sum_{j=1}^{l_{2}} \sum_{s=m_{0}}^{M-1} \sum_{t=n_{0}}^{N-1}\left[\bar{f}_{j}(s, t, m, n) \frac{h_{j}}{p} K_{3}^{\left(h_{j}-p\right) / p}+\bar{g}_{j}(s, t, m, n) \frac{v_{j}}{p} K_{4}^{\left(v_{j}-p\right) / p}\right] b(s, t) \bar{C}(s, t) .
\end{aligned}
$$

Proof. Given $b(m, n)=\max \left\{b_{1}(m, n), b_{2}(m, n)\right\}$, for $(m, n) \in$ $\Omega$, we have

$$
\begin{array}{cc}
u^{p}(m, n) \leq a(m, n)+b(m, n) & \left.+g_{j}(s, t, m, n) H_{j}\left(s, t, u^{v_{j}}(s, t)\right)+w_{j}(s, t, m, n)\right] . \\
\cdot \sum_{i=1}^{l_{1}} \sum_{s=m_{0}}^{m-1} \sum_{t=n_{0}}^{n-1}\left[c_{i}(s, t, m, n) u^{q_{i}}(s, t)\right. & \text { Define function } \bar{v}(m, n) \text { by } \\
\left.+d_{i}(s, t, m, n) u^{r_{i}}(s, t)+e_{i}(s, t, m, n)\right]+b(m, n) & \\
\bar{v}(m, n)=\sum_{i=1}^{l_{1}} \sum_{s=m_{0}}^{m-1} \sum_{t=n_{0}}^{n-1}\left[c_{i}(s, t, m, n) u^{q_{i}}(s, t)+d_{i}(s, t, m, n) u^{r_{i}}(s, t)+e_{i}(s, t, m, n)\right] \\
+\sum_{j=1}^{l_{2}} \sum_{s=m_{0}}^{M-1} \sum_{t=n_{0}}^{N-1}\left[f_{j}(s, t, m, n) H_{j}\left(s, t, u^{h_{j}}(s, t)\right)+g_{j}(s, t, m, n) H_{j}\left(s, t, u^{v_{j}}(s, t)\right)+w_{j}(s, t, m, n)\right] .
\end{array}
$$

$$
\cdot \sum_{j=1}^{l_{2}} \sum_{s=m_{0}}^{M-1} \sum_{t=n_{0}}^{N-1}\left[f_{j}(s, t, m, n) H_{j}\left(s, t, u^{h_{j}}(s, t)\right)\right.
$$

Then or

$$
u(m, n) \leq(a(m, n)+b(m, n) \bar{v}(m, n))^{1 / p} .
$$


By Lemma 3, we have

$$
\begin{aligned}
& \bar{v}(m, n) \leq \sum_{i=1}^{l_{1}} \sum_{s=m_{0}}^{m-1} \sum_{t=n_{0}}^{n-1}\left\{c_{i}(s, t, m, n)(a(s, t)+b(s, t) \bar{v}(s, t))^{q_{i} / p}\right. \\
& \left.+d_{i}(s, t, m, n)(a(s, t)+b(s, t) \bar{v}(s, t))^{r_{i} / p}+e_{i}(s, t, m, n)\right\} \\
& +\sum_{j=1}^{l_{2}} \sum_{s=m_{0}}^{M-1} \sum_{t=n_{0}}^{N-1}\left\{f_{j}(s, t, m, n) H_{j}\left(s, t,(a(s, t)+b(s, t) \bar{v}(s, t))^{h_{j} / p}\right)\right. \\
& \left.+g_{j}(s, t, m, n) H_{j}\left(s, t,(a(s, t)+b(s, t) \bar{v}(s, t))^{v_{j} / p}\right)+w_{j}(s, t, m, n)\right\} \\
& \leq \sum_{i=1}^{l_{1}} \sum_{s=m_{0}}^{m-1} \sum_{t=n_{0}}^{n-1}\left\{c_{i}(s, t, m, n)\left[\frac{q_{i}}{p} K_{1}^{\left(q_{i}-p\right) / p}(a(s, t)+b(s, t) \bar{v}(s, t))+\frac{p-q_{i}}{p} K_{1}^{q_{i} / p}\right]\right. \\
& \left.+d_{i}(s, t, m, n)\left[\frac{r_{i}}{p} K_{2}^{\left(r_{i}-p\right) / p}(a(s, t)+b(s, t) \bar{v}(s, t))+\frac{p-r_{i}}{p} K_{2}^{r_{i} / p}\right]+e_{i}(s, t, m, n)\right\} \\
& +\sum_{j=1}^{l_{2}} \sum_{s=m_{0}}^{M-1} \sum_{t=n_{0}}^{N-1}\left\{f _ { j } ( s , t , m , n ) \left[H_{j}\left(s, t, \frac{h_{j}}{p} K_{3}^{\left(h_{j}-p\right) / p}(a(s, t)+b(s, t) \bar{v}(s, t))+\frac{p-h_{j}}{p} K_{3}^{h_{j} / p}\right)\right.\right. \\
& \left.-H_{j}\left(s, t, \frac{h_{j}}{p} K_{3}^{\left(h_{j}-p\right) / p} a(s, t)+\frac{p-h_{j}}{p} K_{3}^{h_{j} / p}\right)+H_{j}\left(s, t, \frac{h_{j}}{p} K_{3}^{\left(h_{j}-p\right) / p} a(s, t)+\frac{p-h_{j}}{p} K_{3}^{h_{j} / p}\right)\right]+g_{j}(s, t, m, n) \\
& \cdot\left[H_{j}\left(s, t, \frac{v_{j}}{p} K_{4}^{\left(v_{j}-p\right) / p}(a(s, t)+b(s, t) \bar{v}(s, t))+\frac{p-v_{j}}{p} K_{4}^{v_{j} / p}\right)-H_{j}\left(s, t, \frac{v_{j}}{p} K_{4}^{\left(v_{j}-p\right) / p} a(s, t)+\frac{p-v_{j}}{p} K_{4}^{v_{j} / p}\right)\right. \\
& \left.\left.+H_{j}\left(s, t, \frac{v_{j}}{p} K_{4}^{\left(v_{j}-p\right) / p} a(s, t)+\frac{p-v_{j}}{p} K_{4}^{v_{j} / p}\right)\right]+w_{j}(s, t, m, n)\right\} \\
& \leq \sum_{i=1}^{l_{1}} \sum_{s=m_{0}}^{m-1} \sum_{t=n_{0}}^{n-1}\left\{c_{i}(s, t, m, n)\left[\frac{q_{i}}{p} K_{1}^{\left(q_{i}-p\right) / p}(a(s, t)+b(s, t) \bar{v}(s, t))+\frac{p-q_{i}}{p} K_{1}^{q_{i} / p}\right]\right. \\
& \left.+d_{i}(s, t, m, n)\left[\frac{r_{i}}{p} K_{2}^{\left(r_{i}-p\right) / p}(a(s, t)+b(s, t) \bar{v}(s, t))+\frac{p-r_{i}}{p} K_{2}^{r_{i} / p}\right]+e_{i}(s, t, m, n)\right\} \\
& +\sum_{j=1}^{l_{2}} \sum_{s=m_{0}}^{M-1} \sum_{t=n_{0}}^{N-1}\left\{f _ { j } ( s , t , m , n ) \left[L_{j}\left(s, t, \frac{h_{j}}{p} K_{3}^{\left(h_{j}-p\right) / p} a(s, t)+\frac{p-h_{j}}{p} K_{3}^{h_{j} / p}\right)\right.\right. \\
& \left.\cdot \frac{h_{j}}{p} K_{3}^{\left(h_{j}-p\right) / p} b(s, t) \bar{v}(s, t)+H_{j}\left(s, t, \frac{h_{j}}{p} K_{3}^{\left(h_{j}-p\right) / p} a(s, t)+\frac{p-h_{j}}{p} K_{3}^{h_{j} / p}\right)\right]+g_{j}(s, t, m, n)\left[L _ { j } \left(s, t, \frac{v_{j}}{p} K_{4}^{\left(v_{j}-p\right) / p} a(s, t)\right.\right. \\
& \left.\left.\left.+\frac{p-v_{j}}{p} K_{4}^{v_{j} / p}\right) \frac{v_{j}}{p} K_{4}^{\left(v_{j}-p\right) / p} b(s, t) \bar{v}(s, t)+H_{j}\left(s, t, \frac{v_{j}}{p} K_{4}^{\left(v_{j}-p\right) / p} a(s, t)+\frac{p-v_{j}}{p} K_{4}^{v_{j} / p}\right)\right]+w_{j}(s, t, m, n)\right\}=\bar{R}(m, n) \\
& +\sum_{i=1}^{l_{1}} \sum_{s=m_{0}}^{m-1} \sum_{t=n_{0}}^{n-1}\left[c_{i}(s, t, m, n) \frac{q_{i}}{p} K_{1}^{\left(q_{i}-p\right) / p}+d_{i}(s, t, m, n) \frac{r_{i}}{p} K_{2}^{\left(r_{i}-p\right) / p}\right] b(s, t) \bar{v}(s, t),
\end{aligned}
$$

where 


$$
\bar{R}(m, n)=\bar{J}(m, n)+\sum_{j=1}^{l_{2}} \sum_{s=m_{0}}^{M-1} \sum_{t=n_{0}}^{N-1}\left[\bar{f}_{j}(s, t, m, n) \frac{h_{j}}{p} K_{3}^{\left(h_{j}-p\right) / p}+\bar{g}_{j}(s, t, m, n) \frac{v_{j}}{p} K_{4}^{\left(v_{j}-p\right) / p}\right] b(s, t) \bar{v}(s, t),
$$

and $\bar{J}(m, n), \bar{f}_{j}(s, t, m, n), \bar{g}_{j}(s, t, m, n)$ are defined in (54)(56).

Similar to the process of (17)-(23), we get

$$
\bar{v}(m, n) \leq \frac{\bar{J}(M, N)}{1-\bar{\lambda}(M, N)} \bar{C}(m, n),
$$

where $\bar{C}(m, n), \bar{\lambda}(m, n)$ are defined in (52) and (57).
Combining (61) and (64), we get the desired result.

Theorem 9. Let $\varphi(m, n) \in \wp_{+}(\Omega), u(m, n), a(m, n), b_{1}(m, n)$, $b_{2}(m, n), c_{i}(s, t, m, n), d_{i}(s, t, m, n), e_{i}(s, t, m, n), f_{j}(s, t, m, n)$, $g_{j}(s, t, m, n), w_{j}(s, t, m, n), p, q_{i}, r_{i}, h_{j}, v_{j}$ be defined as in Theorem 4. Assume that $a(m, n)$ is nondecreasing in the first variable. $H_{j}, L_{j}\left(j=1,2, \ldots, l_{2}\right)$ are defined as in Theorem 7 . If

$$
\begin{aligned}
u^{p}(m, n) \leq & a(m, n)+\sum_{s=m_{0}}^{m-1} \varphi(s, n) u^{p}(s, n) \\
& +b_{1}(m, n) \sum_{i=1}^{l_{1}} \sum_{s=m_{0}}^{m-1} \sum_{t=n_{0}}^{n-1}\left[c_{i}(s, t, m, n) u^{q_{i}}(s, t)+d_{i}(s, t, m, n) u^{r_{i}}(s, t)+e_{i}(s, t, m, n)\right] \\
& +b_{2}(m, n) \sum_{j=1}^{l_{2}} \sum_{s=m_{0}}^{M-1} \sum_{t=n_{0}}^{N-1}\left[f_{j}(s, t, m, n) H_{j}\left(s, t, u^{h_{j}}(s, t)\right)+g_{j}(s, t, m, n) H_{j}\left(s, t, u^{v_{j}}(s, t)\right)+w_{j}(s, t, m, n)\right],
\end{aligned}
$$

then, for $(m, n) \in \Omega$, we have provided that $\widehat{\lambda}(M, N)<1$, where

$u(m, n)$

$$
\leq\left\{a(m, n)+b(m, n) \frac{\widehat{J}(M, N)}{1-\widehat{\lambda}(M, N)} \widehat{C}(m, n)\right\}^{1 / p}
$$

$$
\begin{aligned}
& \widehat{\varphi}(m, n)=\prod_{s=m_{0}}^{m-1}[1+\varphi(s, n)], \\
& b(m, n)=\max \left\{b_{1}(m, n), b_{2}(m, n)\right\} \\
& \widehat{C}(m, n)=\exp \left\{\sum_{s=m_{0}}^{m-1} \sum_{t=n_{0}}^{n-1} \widehat{B}(s, t, m, n)\right\} \\
& \widehat{B}(s, t, m, n)=\sum_{i=1}^{l_{1}}\left[\widehat{c}_{i}(s, t, m, n) \frac{q_{i}}{p} K_{1}^{\left(q_{i}-p\right) / p}+\widehat{d}_{i}(s, t, m, n) \frac{r_{i}}{p} K_{2}^{\left(r_{i}-p\right) / p}\right] b(s, t) \\
& \widehat{J}(m, n)=\sum_{i=1}^{l_{1}} \sum_{s=m_{0}}^{m-1} \sum_{t=n_{0}}^{n-1}\left\{\widehat{c}_{i}(s, t, m, n)\left[\frac{q_{i}}{p} K_{1}^{\left(q_{i}-p\right) / p} a(s, t)+\frac{p-q_{i}}{p} K_{1}^{q_{i} / p}\right]\right. \\
& \left.\quad+\widehat{d}_{i}(s, t, m, n)\left[\frac{r_{i}}{p} K_{2}^{\left(r_{i}-p\right) / p} a(s, t)+\frac{p-r_{i}}{p} K_{2}^{r_{i} / p}\right]+e_{i}(s, t, m, n)\right\} \\
& \quad+\sum_{j=1}^{l_{2}} \sum_{s=m_{0}}^{M-1} \sum_{t=n_{0}}^{N-1}\left\{f_{\mathrm{j}}(s, t, m, n) H_{j}\left[s, t,(\widehat{\varphi}(s, t))^{h_{j} / p}\left(\frac{h_{j}}{p} K_{3}^{\left(h_{j}-p\right) / p} a(s, t)+\frac{p-h_{j}}{p} K_{3}^{h_{j} / p}\right)\right]\right. \\
& \left.\quad+g_{j}(s, t, m, n) H_{j}\left[s, t,(\widehat{\varphi}(s, t))^{v_{j} / p}\left(\frac{v_{j}}{p} K_{4}^{\left(v_{j}-p\right) / p} a(s, t)+\frac{p-v_{j}}{p} K_{4}^{v_{j} / p}\right)\right]+w_{j}(s, t, m, n)\right\}
\end{aligned}
$$




$$
\begin{aligned}
& \widehat{\lambda}(m, n)=\sum_{j=1}^{l_{2}} \sum_{s=m_{0}}^{M-1} \sum_{\mathrm{t}=n_{0}}^{N-1}\left[\widehat{f}_{j}(s, t, m, n) \frac{h_{j}}{p} K_{3}^{\left(h_{j}-p\right) / p}+\widehat{g}_{j}(s, t, m, n) \frac{v_{j}}{p} K_{4}^{\left(v_{j}-p\right) / p}\right] b(s, t) \widehat{C}(s, t), \\
& \widetilde{c}_{i}(s, t, m, n)=c_{i}(s, t, m, n)(\widehat{\varphi}(s, t))^{q_{i} / p}, \\
& \tilde{d}_{i}(s, t, m, n)=d_{i}(s, t, m, n)(\widehat{\varphi}(s, t))^{r_{i} / p}, \quad i=1,2, \ldots, l_{1}, \\
& \widehat{f}_{j}(s, t, m, n)=f_{j}(s, t, m, n)(\widehat{\varphi}(s, t))^{h_{j} / p} L_{j}\left[s, t,(\widehat{\varphi}(s, t))^{h_{j} / p}\left(\frac{h_{j}}{p} K_{3}^{\left(h_{j}-p\right) / p} a(s, t)+\frac{p-h_{j}}{p} K_{3}^{h_{j} / p}\right)\right], \\
& \widehat{g}_{j}(s, t, m, n)=g_{j}(s, t, m, n)(\widehat{\varphi}(s, t))^{v_{j} / p} L_{j}\left[s, t,(\widehat{\varphi}(s, t))^{v_{j} / p}\left(\frac{v_{j}}{p} K_{4}^{\left(v_{j}-p\right) / p} a(s, t)+\frac{p-v_{j}}{p} K_{4}^{v_{j} / p}\right)\right],
\end{aligned}
$$$$
j=1,2, \ldots, l_{2} \text {. }
$$

The proof for Theorem 9 is similar to the combination of Theorems 7 and 8 , and we omit the details here.

\section{Applications}

In this section, we will present some applications for the established results to study boundedness, uniqueness, and continuous dependence of solutions of certain difference equations.

Consider the following Volterra-Fredholm sum-difference equations:

$$
\begin{aligned}
& u^{p}(m, n)=a(m, n)+\sum_{s=m_{0}}^{m-1} \sum_{t=n_{0}}^{n-1}[C(s, t, m, n, u(s, t)) \\
& +D(s, t, m, n, u(s, t))+E(s, t, m, n)] \\
& +\sum_{s=m_{0}}^{M-1} \sum_{t=n_{0}}^{N-1}[F(s, t, m, n, u(s, t)) \\
& +G(s, t, m, n, u(s, t))+W(s, t, m, n)],
\end{aligned}
$$

where $u(m, n), a(m, n) \in \wp(\Omega), p \geq 1$ is an odd number, $C, D, F, G: \Omega^{2} \times R \rightarrow R, E, W \in \wp\left(\Omega^{2}\right)$.

Theorem 10. Assume that functions $C, D, E, F, G, W$ in equation (68) satisfy the following conditions:

$$
\begin{gathered}
\left|C\left(s, t, m, n, u_{1}\right)\right| \leq c_{1}(s, t, m, n)\left|u_{1}^{q}\right|, \\
\left|D\left(s, t, m, n, u_{1}\right)\right| \leq d_{1}(s, t, m, n)\left|u_{1}^{r}\right|, \\
|E(s, t, m, n)| \leq e_{1}(s, t, m, n), \\
\left|F\left(s, t, m, n, u_{1}\right)\right| \leq f_{1}(s, t, m, n)\left|u_{1}^{h}\right|, \\
\left|G\left(s, t, m, n, u_{1}\right)\right| \leq g_{1}(s, t, m, n)\left|u_{1}^{v}\right|, \\
|W(s, t, m, n)| \leq w_{1}(s, t, m, n)
\end{gathered}
$$

for $(m, n) \in \Omega, u_{1} \in R$, where $q, r, h, v$ are nonnegative constants satisfying $p \geq q>0, p \geq r>0, p \geq h>0, p \geq v>0$, $c_{1}, d_{1}, e_{1}, f_{1}, g_{1}, w_{1} \in \wp_{+}\left(\Omega^{2}\right)$ which are nondecreasing in the last two variables; then one has

$|u(m, n)|$

$$
\leq\left\{|a(m, n)|+\frac{J_{1}(M, N)}{1-\lambda_{1}(M, N)} C_{1}(m, n)\right\}^{1 / p},
$$

provided that $\lambda_{1}(M, N)<1$, where

$$
\begin{aligned}
& C_{1}(m, n)=\exp \left\{\sum_{s=m_{0}}^{m-1} \sum_{t=n_{0}}^{n-1} B_{1}(s, t, m, n)\right\}, \\
& B_{1}(s, t, m, n)=c_{1}(s, t, m, n) \frac{q}{p} K_{1}^{(q-p) / p}+d_{1}(s, t, m, n) \\
& \quad \cdot \frac{r}{p} K_{2}^{(r-p) / p}, \\
& J_{1}(m, n)=\sum_{s=m_{0}} \sum_{t=n_{0}}^{m-1}\left\{c_{1}(s, t, m, n)\right. \\
& \quad \cdot\left[\frac{q}{p} K_{1}^{(q-p) / p}|a(s, t)|+\frac{p-q}{p} K_{1}^{q / p}\right] \\
& +d_{1}(s, t, m, n)\left[\frac{r}{p} K_{2}^{(r-p) / p}|a(s, t)|+\frac{p-r}{p} K_{2}^{r / p}\right] \\
& \left.+e_{1}(s, t, m, n)\right\}+\sum_{s=m_{0}}^{M-1} \sum_{t=n_{0}}^{N-1}\left\{f_{1}(s, t, m, n)\right. \\
& \quad \cdot\left[\frac{h}{p} K_{3}^{(h-p) / p}|a(s, t)|+\frac{p-h}{p} K_{3}^{h / p}\right] \\
& +g_{1}(s, t, m, n)\left[\frac{v}{p} K_{4}^{(v-p) / p}|a(s, t)|+\frac{p-v}{p} K_{4}^{v / p}\right] \\
& \left.+w_{1}(s, t, m, n)\right\},
\end{aligned}
$$




$$
\begin{gathered}
\lambda_{1}(m, n)=\sum_{s=m_{0}}^{M-1} \sum_{t=n_{0}}^{N-1}\left[f_{1}(s, t, m, n) \frac{h}{p} K_{3}^{(h-p) / p}\right. \\
\left.+g_{1}(s, t, m, n) \frac{v}{p} K_{4}^{(v-p) / p}\right] C_{1}(s, t) .
\end{gathered}
$$

Proof. Using conditions (69) to (68), we have

$$
\begin{aligned}
& \left|u^{p}(m, n)\right| \leq|a(m, n)| \\
& +\sum_{s=m_{0}} \sum_{t=n_{0}}^{m-1}[|C(s, t, m, n, u(s, t))| \\
& +|D(s, t, m, n, u(s, t))|+|E(s, t, m, n)|] \\
& +\sum_{s=m_{0}} \sum_{t=n_{0}}^{M-1}[|F(s, t, m, n, u(s, t))| \\
& +|G(s, t, m, n, u(s, t))|+|W(s, t, m, n)|] \leq \mid a(m, \\
& +n) \mid+\sum_{s=m_{0}}^{N-1} \sum_{t=n_{0}}^{n-1}\left[c_{1}(s, t, m, n)\left|u^{q}(s, t)\right|\right. \\
& \left.+d_{1}(s, t, m, n)\left|u^{r}(s, t)\right|+e_{1}(s, t, m, n)\right] \\
& +\sum_{s=m_{0}}^{M-1} \sum_{t=n_{0}}^{N-1}\left[f_{1}(s, t, m, n)\left|u^{h}(s, t)\right|\right. \\
& \left.+g_{1}(s, t, m, n)\left|u^{v}(s, t)\right|+w_{1}(s, t, m, n)\right] .
\end{aligned}
$$

Then a suitable application of Theorem 4 (with $l_{1}=l_{2}=1$ ) to (72) yields the desired result.

The following theorem deals with the uniqueness of the solutions of (68).

Theorem 11. Supposing that

$$
\begin{aligned}
& \left|C\left(s, t, m, n, u_{1}\right)-C\left(s, t, m, n, u_{2}\right)\right| \\
& \quad \leq c_{1}(s, t, m, n)\left|u_{1}^{p}-u_{2}^{p}\right|, \\
& \left|F\left(s, t, m, n, u_{1}\right)-F\left(s, t, m, n, u_{2}\right)\right| \\
& \quad \leq f_{1}(s, t, m, n)\left|u_{1}^{p}-u_{2}^{p}\right|, \\
& \left|D\left(s, t, m, n, u_{1}\right)-D\left(s, t, m, n, u_{2}\right)\right| \\
& \quad \leq d_{1}(s, t, m, n)\left|u_{1}^{p}-u_{2}^{p}\right|, \\
& \left|G\left(s, t, m, n, u_{1}\right)-G\left(s, t, m, n, u_{2}\right)\right| \\
& \quad \leq g_{1}(s, t, m, n)\left|u_{1}^{p}-u_{2}^{p}\right|
\end{aligned}
$$

hold for $u_{1}, u_{2} \in R$, where $c_{1}, d_{1}, f_{1}, g_{1} \in \wp_{+}\left(\Omega^{2}\right)$ are nondecreasing in the last two variables,

$$
\lambda(M, N)
$$$$
=\sum_{s=m_{0}}^{M-1} \sum_{t=n_{0}}^{N-1}\left[f_{1}(s, t, M, N)+g_{1}(s, t, M, N)\right] C(s, t)
$$

$<1$,

$$
B(s, t, m, n)=c_{1}(s, t, m, n)+d_{1}(s, t, m, n),
$$

$$
C(s, t)=\exp \left\{\sum_{s=m_{0}}^{m-1} \sum_{t=n_{0}}^{n-1} B(s, t, m, n)\right\},
$$

then (68) has at most one solution.

Proof. Assume that $u(m, n), \bar{u}(m, n)$ are two solutions of (68). Then

$$
\begin{aligned}
& \left|u^{p}(m, n)-\bar{u}^{p}(m, n)\right| \\
& \quad \leq \sum_{s=m_{0}}^{m-1} \sum_{t=n_{0}}^{n-1}[|C(s, t, m, n, u(s, t))-C(s, t, m, n, \bar{u}(s, t))| \\
& +|D(s, t, m, n, u(s, t))-D(s, t, m, n, \bar{u}(s, t))|] \\
& \quad+\sum_{s=m_{0}}^{M-1} \sum_{t=n_{0}}^{N-1}[|F(s, t, m, n, u(s, t))-F(s, t, m, n, \bar{u}(s, t))| \\
& +|G(s, t, m, n, u(s, t))-G(s, t, m, n, \bar{u}(s, t))|] \\
& \quad \leq \sum_{s=m_{0}}^{m-1} \sum_{t=n_{0}}^{n-1}\left[c_{1}(s, t, m, n)+d_{1}(s, t, m, n)\right] \mid u^{p}(s, t) \\
& \quad-\bar{u}^{p}(s, t) \mid+\sum_{s=m_{0}}^{M-1} \sum_{t=n_{0}}^{N-1}\left[f_{1}(s, t, m, n)+g_{1}(s, t, m, n)\right] \\
& \quad|| u^{p}(s, t)-\bar{u}^{p}(s, t) \mid .
\end{aligned}
$$

Treat $\left|u^{p}(m, n)-\bar{u}^{p}(m, n)\right|$ as one variable, and a suitable application of Corollary 6 yields $\left|u^{p}(m, n)-\bar{u}^{p}(m, n)\right| \leq 0$, which implies that $u^{p}(m, n) \equiv \bar{u}^{p}(m, n)$. Since $p$ is an odd number, then we have $u^{p}(m, n)=\bar{u}^{p}(m, n)$, and the proof is complete.

Finally we study the continuous dependence of the solutions of (68) on functions $a, C, D, E, F, G, W$. For this, we consider the following variation of (68):

$$
\begin{aligned}
& \widetilde{u}^{p}(m, n)=\widetilde{a}(m, n)+\sum_{s=m_{0}} \sum_{t=n_{0}}^{n-1}[\widetilde{C}(s, t, m, n, \widetilde{u}(s, t)) \\
& +\widetilde{D}(s, t, m, n, \widetilde{u}(s, t))+\widetilde{E}(s, t, m, n)] \\
& +\sum_{s=m_{0}} \sum_{t=n_{0}}^{M-1}[\widetilde{F}(s, t, m, n, \widetilde{u}(s, t)) \\
& +\widetilde{G}(s, t, m, n, \widetilde{u}(s, t))+\widetilde{W}(s, t, m, n)]
\end{aligned}
$$


where $\widetilde{C}, \widetilde{D}, \widetilde{F}, \widetilde{G}: \Omega^{2} \times R \rightarrow R, \widetilde{E}, \widetilde{W} \in \wp\left(\Omega^{2}\right)$ and $p \geq 1$ is an odd number.

Theorem 12. Consider (68) and (76). If

$$
\begin{aligned}
& \left|C\left(s, t, m, n, u_{1}(s, t)\right)-C\left(s, t, m, n, u_{2}(s, t)\right)\right| \\
& \quad \leq c_{1}(s, t, m, n)\left|u_{1}^{p}-u_{2}^{p}\right|, \\
& \left|D\left(s, t, m, n, u_{1}(s, t)\right)-D\left(s, t, m, n, u_{2}(s, t)\right)\right| \\
& \quad \leq d_{1}(s, t, m, n)\left|u_{1}^{p}-u_{2}^{p}\right|, \\
& \left|F\left(s, t, m, n, u_{1}(s, t)\right)-F\left(s, t, m, n, u_{2}(s, t)\right)\right| \\
& \quad \leq f_{1}(s, t, m, n)\left|u_{1}^{p}-u_{2}^{p}\right|, \\
& \left|G\left(s, t, m, n, u_{1}(s, t)\right)-G\left(s, t, m, n, u_{2}(s, t)\right)\right| \\
& \quad \leq g_{1}(s, t, m, n)\left|u_{1}^{p}-u_{2}^{p}\right|,
\end{aligned}
$$

hold for $u_{1}, u_{2} \in R$, where $c_{1}, d_{1}, f_{1}, g_{1} \in \wp_{+}\left(\Omega^{2}\right)$, and are nondecreasing in the last two variables, furthermore, for all solution $\tilde{u}$ of (76), the following conditions hold for $(m, n) \in \Omega$ :

$$
\begin{array}{r}
|a(m, n)-\widetilde{a}(m, n)| \leq \frac{\varepsilon}{4}, \\
\sum_{s=m_{0}} \sum_{t=n_{0}}^{m-1}|E(s, t, m, n)-\widetilde{E}(s, t, m, n)| \leq \frac{\varepsilon}{8}, \\
\sum_{s=m_{0}}^{M-1} \sum_{t=n_{0}}^{N-1}|W(s, t, m, n)-\widetilde{W}(s, t, m, n)| \leq \frac{\varepsilon}{8}, \\
\sum_{s=m_{0}}^{m-1} \sum_{t=n_{0}}^{n-1}|F(s, t, m, n, \widetilde{u})-\widetilde{F}(s, t, m, n, \widetilde{u})| \leq \frac{\varepsilon}{8}, \\
\sum_{s=m_{0}}^{M-1} \sum_{t=n_{0}}^{N-1}|C(s, t, m, n, \widetilde{u})-\widetilde{C}(s, t, m, n, \widetilde{u})| \leq \frac{\varepsilon}{8}, \\
\sum_{s=m_{0}}^{m-1} \sum_{t=n_{0}}^{n-1}|D(s, t, m, n, \widetilde{u})-\widetilde{D}(s, t, m, n, \widetilde{u})| \leq \frac{\varepsilon}{8}, \\
\sum_{s=m_{0}}^{M-1} \sum_{t=n_{0}}^{N-1}|G(s, t, m, n, \widetilde{u})-\widetilde{G}(s, t, m, n, \widetilde{u})| \leq \frac{\varepsilon}{8},
\end{array}
$$

where $\varepsilon>0$ is an arbitrary constant. Then

$$
\begin{aligned}
& \left|u^{p}(m, n)-\widetilde{u}^{p}(m, n)\right| \\
& \quad \leq \varepsilon\left[1+\frac{J_{2}(M, N)}{1-\lambda_{2}(M, N)} C_{2}(m, n)\right],
\end{aligned}
$$

where $\lambda_{2}(M, N)<1$, and

$$
\begin{aligned}
& C_{2}(m, n)=\exp \left\{\sum_{s=m_{0}}^{m-1} \sum_{t=n_{0}}^{n-1} B_{2}(s, t, m, n)\right\}, \\
& B_{2}(s, t, m, n)=\left[c_{1}(s, t, m, n)+d_{1}(s, t, m, n)\right], \\
& \lambda_{2}(m, n) \\
& \quad \sum_{s=m_{0}}^{M-1} \sum_{t=n_{0}}^{N-1}\left[f_{1}(s, t, m, n)+g_{1}(s, t, m, n)\right] C_{2}(s, t),
\end{aligned}
$$

$J_{2}(m, n)$

$$
\begin{aligned}
= & \sum_{s=m_{0}}^{m-1} \sum_{t=n_{0}}^{n-1}\left[c_{1}(s, t, m, n)+d_{1}(s, t, m, n)\right] \\
& +\sum_{s=m_{0}}^{M-1} \sum_{t=n_{0}}^{N-1}\left[f_{1}(s, t, m, n)+g_{1}(s, t, m, n)\right]
\end{aligned}
$$

for $(m, n) \in \Omega$. That is, $u^{p}$ depends continuously on the functions $a, C, D, E, F, G, W$.

Proof. Let $u(m, n)$ and $\widetilde{u}(m, n)$ be solutions of (68) and (76), respectively. Then $u(m, n)$ satisfies $(68)$ and $\widetilde{u}(m, n)$ satisfies (76). Hence

$$
\begin{aligned}
& \left|u^{p}(m, n)-\widetilde{u}^{p}(m, n)\right| \leq|a(m, n)-\widetilde{a}(m, n)| \\
& +\sum_{s=m_{0}}^{m-1} \sum_{t=n_{0}}^{n-1}[|C(s, t, m, n, u(s, t))-\widetilde{C}(s, t, m, n, \widetilde{u}(s, t))| \\
& +|D(s, t, m, n, u(s, t))-\widetilde{D}(s, t, m, n, \widetilde{u}(s, t))| \\
& +|E(s, t, m, n)-\widetilde{E}(s, t, m, n)|] \\
& +\sum_{s=m_{0}}^{M-1} \sum_{t=n_{0}}^{N-1}[|F(s, t, m, n, u(s, t))-\widetilde{F}(s, t, m, n, \widetilde{u}(s, t))| \\
& +|G(s, t, m, n, u(s, t))-\widetilde{G}(s, t, m, n, \widetilde{u}(s, t))| \\
& +|W(s, t, m, n)-\widetilde{W}(s, t, m, n)|] \leq \mid a(m, n)-\widetilde{u}(m, \\
& \quad n) \mid \\
& +\sum_{s=m_{0}}^{m-1} \sum_{t=n_{0}}^{n-1}[|C(s, t, m, n, u(s, t))-C(s, t, m, n, \widetilde{u}(s, t))| \\
& +|C(s, t, m, n, \widetilde{u}(s, t))-\widetilde{C}(s, t, m, n, \widetilde{u}(s, t))| \\
& +|D(s, t, m, n, u(s, t))-D(s, t, m, n, \widetilde{u}(s, t))| \\
& +|D(s, t, m, n, \widetilde{u}(s, t))-\widetilde{D}(s, t, m, n, \widetilde{u}(s, t))| \\
& +|E(s, t, m, n)-\widetilde{E}(s, t, m, n)|] \\
& +\sum_{s=m_{0}}^{M-1} \sum_{t=n_{0}}^{N-1}[|F(s, t, m, n, u(s, t))-F(s, t, m, n, \widetilde{u}(s, t))| \\
& +|F(s, t, m, n, \widetilde{u}(s, t))-\widetilde{F}(s, t, m, n, \widetilde{u}(s, t))|
\end{aligned}
$$




$$
\begin{aligned}
& +|G(s, t, m, n, u(s, t))-G(s, t, m, n, \widetilde{u}(s, t))| \\
& +|G(s, t, m, n, \widetilde{u}(s, t))-\widetilde{G}(s, t, m, n, \widetilde{u}(s, t))| \\
& +|W(s, t, m, n)-\widetilde{W}(s, t, m, n)|] \leq \varepsilon \\
& +\sum_{s=m_{0}} \sum_{t=n_{0}}^{m-1}\left[c_{1}(s, t, m, n)+d_{1}(s, t, m, n)\right]\left[u^{p}-\widetilde{u}^{p}\right] \\
& +\sum_{s=m_{0}}^{M-1} \sum_{t=n_{0}}^{N-1}\left[f_{1}(s, t, m, n)+g_{1}(s, t, m, n)\right]\left|u^{p}-\widetilde{u}^{p}\right| .
\end{aligned}
$$

Treat $\left|u^{p}(m, n)-\widetilde{u}^{p}(m, n)\right|$ as one variable, and a suitable application of Corollary 6 (with $l_{1}=l_{2}=1$ ) yields the desired result (79). Hence $u^{p}$ depends continuously on $a, C, D, E$, $F, G, W$.

\section{Conclusions}

The author carried out some new Volterra-Fredholm-type discrete inequalities involving four iterated infinite sums and their corresponding applications. The results are more effective to qualitative analysis of solutions for sum-difference equations, such as the boundedness, uniqueness, and continuous dependence on solutions.

\section{Conflicts of Interest}

The author declares that there are no conflicts of interest.

\section{Acknowledgments}

Run $\mathrm{Xu}$ received a grant from National Science Foundation of China (11671227).

\section{References}

[1] B. Zheng and B. Fu, "Some Volterra-Fredholm type nonlinear discrete inequalities involving four iterated infinite sums," Advances in Difference Equations, vol. 2012, no. 228, 2012.

[2] B. Zheng and Q. Feng, "Some new Volterra-Fredholm-type discrete inequalities and their applications in the theory of difference equations," Abstract and Applied Analysis, vol. 2011, Article ID 584951, 24 pages, 2011.

[3] B. Zheng, "Qualitative and quantitative analysis for solutions to a class of Volterra-Fredholm type difference equation," Advances in Difference Equations, vol. 2011, article no. 30, 2011.

[4] B. G. Pachpatte, "On some fundamental integral inequalities and their discrete analogues," Journal of Inequalities in Pure and Applied Mathematics, vol. 2, no. 2, article 15, 2001.

[5] F. Jiang and F. Meng, "Explicit bounds on some new nonlinear integral inequalities with delay," Journal of Computational and Applied Mathematics, vol. 205, no. 1, pp. 479-486, 2007.

[6] R. Xu and Y. Zhang, "Generalized Gronwall fractional summation inequalities and their applications," Journal of Inequalities and Applications, vol. 2015, no. 42, 2015.

[7] F. W. Meng and D. Ji, "On some new nonlinear discrete inequalities and their applications," Journal of Computational and Applied Mathematics, vol. 208, no. 2, pp. 425-433, 2007.
[8] T. Wang and R. Xu, "Some integral inequalities in two independent variables on time scales," Journal of Mathematical Inequalities, vol. 6, no. 1, pp. 107-118, 2012.

[9] Q. Feng, F. Meng, and B. Fu, "Some new generalized VolterraFredholm type finite difference inequalities involving four iterated sums," Applied Mathematics and Computation, vol. 219, no. 15, pp. 8247-8258, 2013.

[10] Q.-H. Ma and J. Pecaric', "Estimates on solutions of some new nonlinear retarded Volterra-Fredholm type integral inequalities," Nonlinear Analysis, vol. 69, no. 2, pp. 393-407, 2008.

[11] Q.-H. Ma, "Some new nonlinear Volterra-Fredholm-type discrete inequalities and their applications," Journal of Computational and Applied Mathematics, vol. 216, no. 2, pp. 451-466, 2008 .

[12] R. Xu, F. Meng, and C. Song, "On some integral inequalities on time scales and their applications," Journal of Inequalities and Applications, vol. 2010, article 464976, 2010.

[13] L. Wan and R. Xu, "Some generalized integral inequalities and their applications," Journal of Mathematical Inequalities, vol. 7, no. 3, pp. 495-511, 2013.

[14] R. Xu and F. Meng, "Some new weakly singular integral inequalities and their applications to fractional differential equations," Journal of Inequalities and Applications, vol. 2016, no. 78, 2016.

[15] J. Huang and W.-S. Wang, "Some Volterra-Fredholm type nonlinear inequalities involving four iterated infinite integral and application," Journal of Mathematical Inequalities, vol. 10, no. 4, pp. 1105-1118, 2016. 


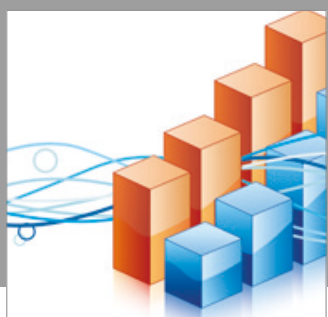

Advances in

Operations Research

vatersals

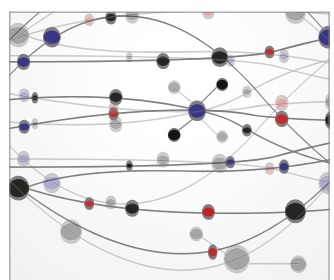

\section{The Scientific} World Journal
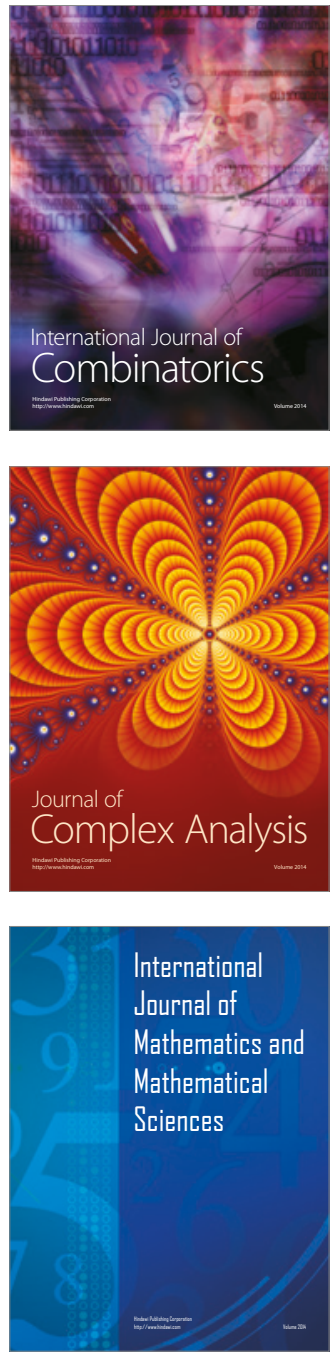
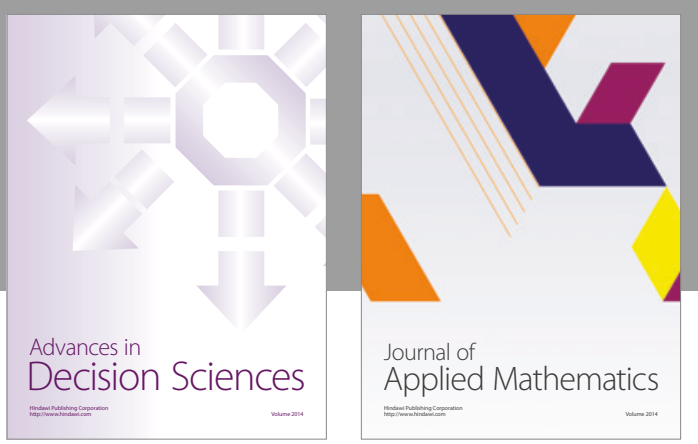

Algebra

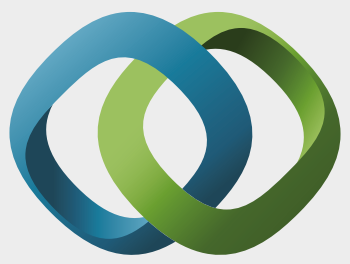

\section{Hindawi}

Submit your manuscripts at

https://www.hindawi.com
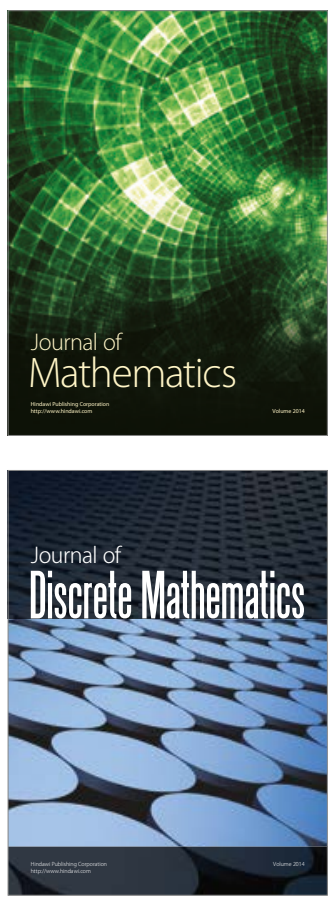

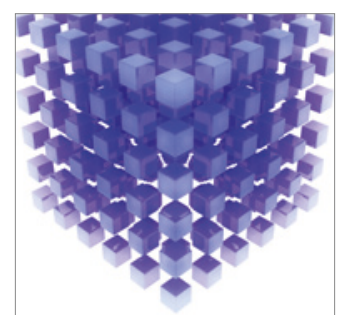

Mathematical Problems in Engineering
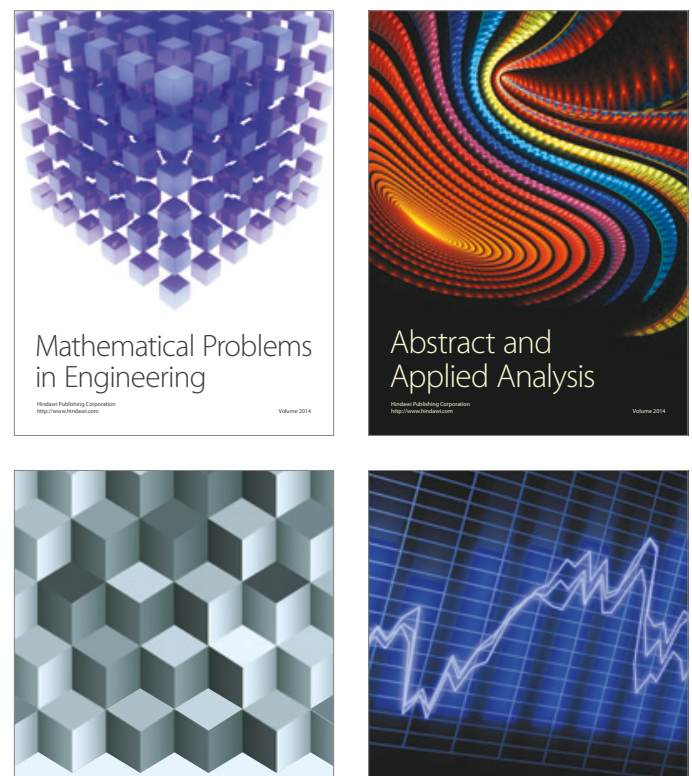

Journal of

Function Spaces

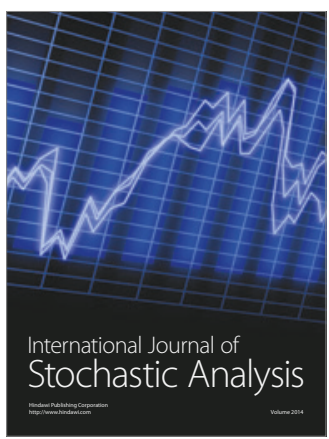

Probability and Statistics
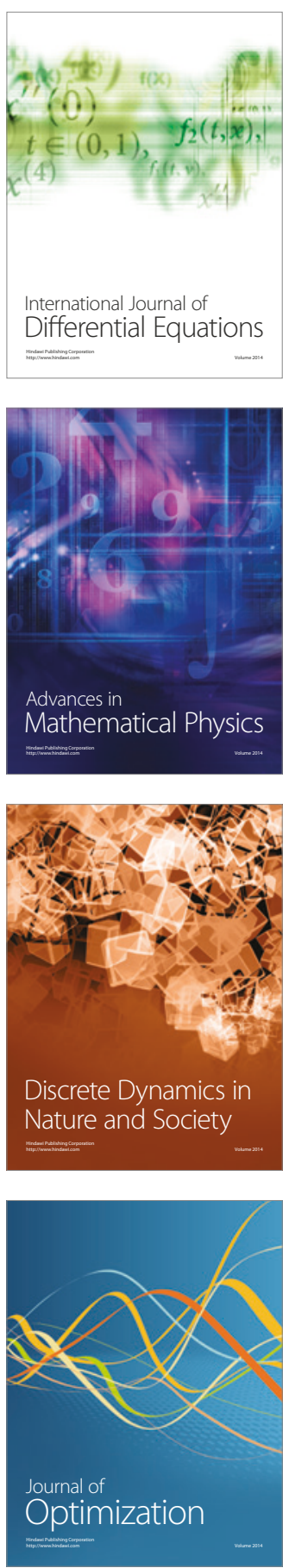